\title{
Screening for cognitive deficits in Parkinson's disease with the Parkinson neuropsychometric dementia assessment (PANDA) instrument
}

\author{
Elke Kalbe ${ }^{\mathrm{a},{ }^{*}}$, Pasquale Calabrese ${ }^{\mathrm{b}}$, Nils Kohn ${ }^{\mathrm{a}}$, Rüdiger Hilker ${ }^{\mathrm{c}}$, Oliver Riedel ${ }^{\mathrm{d}}$, Hans- \\ Ulrich Wittchen ${ }^{\mathrm{d}}$, Richard Dodel ${ }^{\mathrm{e}}$, Jörg Otto ${ }^{\mathrm{f}}$, Georg Ebersbach ${ }^{\mathrm{f}}$, Josef Kessler ${ }^{\mathrm{a}}$
}

\author{
a Department of Neurology, University Hospital, University of Cologne, Germany \\ b Department of Neurology, University Clinic, Bochum, Germany \\ c Department of Neurology, Goethe University Frankfurt, Germany \\ d Institute of Clinical Psychology and Psychotherapy, Technical University of Dresden, Germany \\ e Department of Neurology, Philipps University Marburg, Germany \\ f Neurological Hospital for Movement Disorders, Kliniken Beelitz, Germany
}

\begin{abstract}
Cognitive and affective dysfunctions are frequent but often neglected symptoms in Parkinson's disease (PD). We developed the screening tool Parkinson neuropsychometric dementia assessment (PANDA) with five cognitive tasks and a short depression questionnaire. Healthy subjects and patients without cognitive impairment (PD), mild cognitive disorder (PD-MCD), or dementia (PDD) were examined. The cognition part had a specificity of $91 \%$ and a sensitivity of $90 \%$ for PDD and $77 \%$ for PDD plus PD-MCD patients. The mood questionnaire also had high sensitivity and specificity. We conclude that the PANDA is an economical, easy-to-use and sensitive tool to detect neuropsychological dysfunctions in PD patients in clinical practice.
\end{abstract}

Keywords: Parkinson's disease; Cognitive dysfunction; Dementia; Cognitive screening

\section{Introduction}

Mental dysfunction frequently accompanies the motor symptoms in patients with Parkinson's disease (PD) and ranges from subtle cognitive deficits to dementia. It has been estimated that $20-40 \%$ of PD patients develop dementia in the course of their disease (Parkinson's disease dementia, PDD), and there is recent evidence indicating that the risk of developing dementia increases with disease progression [1] and correlates with the neuropathologic stage in PD [2]. The clinical demarcation of PDD to Lewy body dementia and Alzheimer dementia is often difficult, and the cognitive profile in these patients is inhomogeneous. Although controversial, cognitive disturbances in PDD typically are thought to constitute a form of ,subcortical dementia“ [3] which comprises a progressive dysexecutive syndrome, deficits in attention, memory, and visuospatial abilities. Psychiatric symptoms such as mood disturbances and anxiety are also frequent [4]; depressive symptoms occur in approximately half of PD patients [5]. Although these psychopathological features constitute an important risk factor for caregiver distress, nursing home placement, and decreased quality of life, they are often not considered in a more complex diagnostic decision and the treatment of PD patients [1].

Because the diagnostic assessment of cognitive impairment, dementia, and psychopathological features is usually not feasible in routine care, there is a need for time- 
economic, reliable, sensitive screening tools for cognitive dysfunction. Several cognitive screening instruments exist, for example, the Mini Mental State Examination (MMSE) [6], the Memory Impairment Screen (MIS) [10], the Addenbrooke's Cognitive Examination (ACE) [6], or the Seven Minute Screen [7]. However, these are typically designed for „cortical““ types of dementia such as Alzheimer0s disease which have a neuropsychological profile different from ,subcortical“ dementia [9] and may thus not grasp PD patients' typical cognitive deficits. Furthermore, they usually do not detect dysfunctions from mild deficits up to severe dementia, and none of them accounts for the most frequent neuropsychiatric symptom in PD that may complicate the clinical picture and is important for clinical settings and for general practitioners, namely depression.

On the basis of these considerations we developed a new brief test for PD patients - the Parkinson neuropsychometric dementia assessment instrument (PANDA) highlighting the following requirements: the test should allow for (1) an assessment of cognitive functions typically impaired in PD patients (2) a short (time-economic) and (3) a sensitive assessment of cognitive impairment and dementia in PD, and (4) it should also incorporate a screening of depressive symptoms as the most frequent neuropsychiatric disturbance in PD patients.

In this paper we introduce the PANDA, focusing on (1) the selection of the PANDA's subtests, (2) the description of the PANDA scoring procedures, and (3) the presentation of findings from the normation study with healthy control subjects and PD patients. (4) Furthermore, we compare the performance of the PANDA against the currently most used screening method, the MMSE.

\section{Methods}

\subsection{The PANDA: rationale and selection of subtests}

The PANDA consists of a cognitive test part and a brief mood questionnaire. For the cognition part five tasks which are short and easy to administer and which assess functions typically affected in PD were designed on the basis of established neuropsychological test paradigms. The PANDA was not intended to provide a profile of single functions but rather to have high sensitivity to detect cognitive impairment. Thus, tasks were allowed to include a diversity of domains (e.g. speed of processing, executive function, and attention). These five tasks have been selected from an original number of eight subtests additionally including a Stroop interference task and another visuospatial task (mental rotation). These two tasks were not further considered, however, due to findings in a pilot study with 20 non-demented PD patients and 30 age-matched control subjects. It revealed low sensitivity (correct classification of control subjects) of Stroop task and low specificity (correct classification of the patients) of a mental rotation task (less than 55\%). All tasks of the final PANDA version were well understood and accepted by patients and take approximately 8-10 min to administer including instructions and raw score analysis by the administrator. However, in a few patients with advanced stages of dementia and severe impairment of information processing the administration time went up to 20 min.

(1) Word pair associate learning task with immediate and (subtest 5) delayed recall: Memory impairments are well documented in PD and PDD patients (overview in [1]). In contrast, to learning and memory dysfunction in Alzheimer's disease which are known to be due to primary encoding, storage, and retrieval problems, it is suggested that PD patients' deficits may largely be due to ineffective strategies in encoding and retrieval, that is, executive 
dysfunctions [8], and in fact memory impairment relates to executive function test scores in PDD patients [9].

Word lists with immediate and delayed recall are well-established paradigms to assess verbal memory, and many variants exist [10]. Word pair associate learning tasks impose extraordinary demands on executive functions and attention as two words have to be actively linked to each other by mental strategies. Task difficulty can be varied depending on variables such as frequency or concreteness of single words as well as on semantic association between word pairs.

Following a pilot study in which we tested different numbers of word pairs, recall trials, word characteristics, and relatedness of words, we selected four pairs of concrete, frequent, but semantically unrelated words (banana-suit, paper-frog, storm-ball, feather-scarf) for the PANDA. These pairs are presented three times in the immediate recall condition, each time followed by immediate recall after presentation of one of these words (e.g. banana-?). The order of the words in the recall condition is different in each trial. In the delayed recall condition (subtest 5) approximately 6-8 min after the immediate recall all four word pairs again have to be completed after presentation of one of the words. The number of correctly recalled words is registered. The maximal raw score is 12 for the immediate recall and 4 for the delayed recall condition.

(2) Alternating verbal fluency task: Verbal fluency or word generation tasks are well known paradigms in psychometric testing [10]. They have a very high sensitivity in detecting cognitive impairment and also dementia, even in early stages of the disease [11-13]. This high sensitivity is probably due to the large spectrum of cognitive domains involved including attention and working memory, executive function (especially cognitive flexibility and strategy use), semantic memory (word retrieval), and speed of processing. Several variants of these tasks exist. The main principle is that subjects have to generate words in a restricted time (usually $1 \mathrm{~min}$ ) and with restrictive search conditions, using either words from a semantic category (semantic fluency tasks, e.g. with the category animals), or words beginning with a specific letter (letter fluency tasks, e.g. with the letters F, A, or S), or alternating between categories. PD patients are significantly impaired in verbal fluency tasks, especially of the semantic type [14]. Furthermore, alternating fluency tasks that impose heavy demands upon attention, working memory, and switching ability (i.e. executive functions) may disproportionately be affected [14].

We thus chose an alternating verbal fluency task with two semantic categories (animals and furniture) for the PANDA. Subjects are asked to generate as many examples of these categories as possible within $1 \mathrm{~min}$ and to switch between categories after each item. Both the number of correct words and switching errors are registered.

(3) Visuospatial task: There is considerable evidence of visuospatial dysfunction in PD patients, even when tests contain few motor components [15-18]. Deficits in visual perception may even be among the earliest symptoms of cognitive decline in PD and seem to be independent of the severity of motor dysfunctions or overall intellectual impairment $[19,20]$.

Of two visuospatial tasks originally designed for the PANDA one was sorted out (a mental rotation task) and one was selected for the final test version on the basis of our pilot study with 20 PD patients and 30 control subjects and an analysis of the tasks' sensitivities and specificities. In this latter spatial imagery task which contains three items (after item selection 
out of six original items) half-masked squares with dot patterns are presented and the subject is expected to find the pattern which emerges on removing the mask (see Fig. 1). Items have to be evaluated in the given alignment i.e. they should not be rotated mentally. The maximal raw score is 3 .

(4) Working memory and attention task: Working memory can be defined as the ability to hold and manipulate information by means of one controlling system, the „,central executive“, and additional phonological and visuospatial ,slave“ systems, and its concept is closely linked to the concept of attention [21]. Many studies report working memory dysfunction in PD patients in the verbal (e.g. [22,23]) and nonverbal domain [24] and deficits in attention [25]. Several tests serve to assess working memory and attention. In our pilot study we used two tasks. In the letter-number-sorting task as used by Gold et al. [33] letters and numbers are read aloud in a random order and the patient has to sort letters and numbers separately in their systematic order. In the more simple but still sensitive variant of the task which we finally selected due to results of our pilot study only rows of numbers are presented in a random order (e.g. ,7-2-8-6"), and the subject is expected to repeat these numbers in a systematic order $\left(, 2-6-7-8^{\circ}\right)$. The number of items in the largest row correctly repeated is analyzed. The maximal score is 6 .

Mood questionnaire: We aimed at providing a short and easy assessment of depressive symptoms which are the most common nonmotor symptoms of PD and are a significant cause of functional impairment in these patients [5]. We designed a condensed, mood-related questionnaire with three questions representing central aspects of depressive mood (mood, interest, drive). On a four-stage rating scale the subjects evaluate whether the statements are correct (4 points), mostly correct, mostly incorrect, or incorrect ( 0 points). The maximal score is 9 .

\subsection{Subjects}

One hundred and eight control subjects (control group (CG)) classified as cognitively unimpaired by trained neuropsychologists and with a clinical dementia rating scale score [26] of 0 were included. The control subjects were recruited by placing an advertisement in a local newspaper and a placard in the University Clinic Cologne. Additionally, 124 patients with idiopathic PD (criteria according to the UK Parkinson's Disease Society Brain Bank criteria) [27] were recruited from three specialized Parkinson clinics in Germany. All patients were examined and diagnosed by movement disorders specialists (see Table 1 for Hoehn and Yahr stages). Patients were stratified into clinical PD subtypes (tremor-dominant, equivalent, and akinetic-rigid type) according to the leading clinical symptomatology. The patients were also subdivided into three groups according to their cognitive state: 38 PD patients were diagnosed to have dementia (PDD group) according to DSM-IV criteria [28], 40 patients were classified as having mild cognitive disorder (PD-MCD group) according to ICD-10 [29], and in 46 patients no cognitive impairment was observed (PD group) (Table 1). Despite considerable discussion with regard to the validity of the ICD-10 diagnosis of mild cognitive disorder (MCD) [30] this concept - and not the frequently used concept of mild cognitive impairment (MCI) [31] — was chosen because MCD (1) requires MCI but is not focused on memory dysfunction, well applicable for PD patients, (2) does not demand subjective memory complaints which are not always present in PD patients with mild cognitive dysfunctions, and (3) requires a cerebral disease, damage or dysfunction, or systemic physical disorder as a cause of cognitive dysfunction, which is the case for PD patients. 
All patients underwent elaborate neuropsychological testing by neuropsychologists with different test instruments in the three Parkinson clinics. The evaluation of the cognitive state was made by expert rating on the basis of the results of neuropsychological testing. The PANDA test results were not considered for dementia diagnosis. All procedures were carried out with an adequate understanding and written consent of the subjects.

\subsection{Neuropsychological testing}

All subjects were tested with the PANDA (a final test version can be obtained from the corresponding author). Additionally, the PD patients and also a subpopulation of the $\mathrm{CG}$ ( $\mathrm{n}=$ 50) were examined with the MMSE [32,33] and the Beck Depression Inventory (BDI) [34]. The patients were tested being ,on“.

\subsection{Statistical analysis}

All analyses were carried out using the Statistical Package for the Social Sciences (SPSS) version 14 for Windows (Release 14.0.2, SPSS Inc., Chicago). Non-parametric tests were used to check for differences in distribution (chi-square test) and for analyses of ordinal scale data (Kruskal-Wallis and Mann-Whitney U test). Otherwise, parametric methods were used after checking for statistical normal distribution of the data with the Kolmogorov-Smirnov test, i.e. univariate analysis of variance (ANOVA) with post hoc Scheffé tests and multivariate analyses of covariance (MANCOVA). Multiple comparisons were calculated with Bonferroni adjustment. ROC analyses were performed to define sensitivities and specificities of test scores, optimal cut-off scores, and areas under the curve. Results are indicated as means with standard deviations (SD), or with medians and ranges.

\section{Results}

\subsection{Characteristics of study groups}

Univariate variance analysis revealed significant effects for the factors age $(\mathrm{F}(3,228)=21.1$, $\mathrm{p}<0.001$, partial $\eta 2=0.218)$ and education $(\mathrm{F}(3,228)=10.8, \mathrm{p}<0.001$, partial $\eta 2=0.125)$. Both the CG and the PD group were significantly younger and had more years of education than the PD-MCD and PDD groups. There were no differences in the distribution of gender. The PD subgroups were comparable regarding disease duration, but there was a significant main effect for the Hoehn and Yahr stage (Kruskal-Wallis test, chi-scquare $=18.5, \mathrm{p}<0.001$ ) in that the PDD patients had higher scores than the PD and the PD-MCD group (see Table 1).

\subsection{MMSE and BDI}

The MMSE scores of all study groups and corresponding statistical results are listed in Table 1. A MANCOVA was performed. Age and education for which main group effects had been demonstrated (see Table 1) were included as covariates. There was a significant main effect for the factor study group $(\mathrm{F}(3,168)=49.9, \mathrm{p}<0.001$, partial $\eta 2=0.471)$. Furthermore, there was an effect for education $(F(1,168)=7.7, p<0.05$, partial $\eta 2=0.044)$, but not for age. Group comparisons revealed that the PDD group scored significantly lower than all other study groups $(p<0.001)$. Furthermore group differences were observed for comparison between CG and PD-MCD and PD and PD-MCD $(\mathrm{p}<0.05)$. No other differences were observed. When the MMSE0s original cut-off score for dementia of 24/25 points was used, the instrument had a specificity of $100 \%$ and a sensitivity of $63 \%$. With a higher cut-off of $25 / 26$ which has been proposed to improve the instrument0s diagnostic accuracy to detect 
dementia [35] the specificity remained high at $98 \%$, but the sensitivity was still relatively low at $68 \%$. When both the PDD and the PD-MCD patients were included in the analysis with the higher cutoff score in order to determine the MMSE's general capability to detect cognitive impairment (including mild dysfunction), the sensitivity was only $45 \%$. Contrasting PD and PDD patients with a cut-off score of $25 / 26$ points, the specificity was $100 \%$, and the sensitivity was $68 \%$. An analysis of all PD patients showed that only $28 \%$ of the whole PD group had MMSE scores below this cut-off, although 63\% were actually impaired (either PDMCD or PDD).

The BDI scores of the study groups and corresponding statistical results are listed in Table 1 . In the MANCOVA controlled for the covariates age and education there was a significant main effect for the factor study group $(F(3,168)=9.0, p<0.001$, partial $\eta 2=0.139)$. No effects for age or education were observed. Again, the PDD groups differed from all other study groups in that they had higher depression scores $(p<0.01-p<0.001)$ and PD-MCD patients showed higher depression scores as compared to $C G(p<0.01)$. No other group differences were observed. In total, $39 \%$ of the PD group had BDI scores above the cut-off score for mild to moderate depressive symptoms (11 points or above).

\subsection{PANDA raw scores}

The raw scores of all groups are demonstrated in Fig. 2. To test for group differences in the performance of the PANDA subtests a MANCOVA was calculated with age and education as covariates. This analysis showed significant main effects for the factor study group for all PANDA subtests $(F(3,226)=$ between 17.0 and 44.6 , all $p<0.001$, partial $\eta 2$ between 0.184 and 0.372$)$. There were effects for age in all cognitive PANDA subtests $(F(1,226)$ between 4.3 and 36.2, p between 0.05 and 0.001 , partial $\eta 2$ between 0.019 and 0.138 ). No effects of education were observed. On a single comparison level using Bonferroni adjustment, the only significant differences between the CG and the PD group were revealed in the verbal fluency and spatial imagery tasks $(\mathrm{p}<0.05)$. Both the CG and the PD group scored higher than the PDMCD and the PDD patients in all subtests $(\mathrm{p}<0.05-\mathrm{p}<0.001$ for all group comparisons). The PD-MCD patients reached higher scores than the PDD group in all subtests except for the mental rotation task (all $\mathrm{p}<0.001, \mathrm{p}<0.05$ only for the delayed recall).

\subsection{Transformation of raw scores, determination of the test score, and interpretation of the PANDA}

To receive a meaningful PANDA total score, transformation algorithms for the raw scores had to be defined. First, the verbal fluency task does not have a maximum raw score so that some form of transformation is necessary. Second, in order to receive a highly sensitive instrument the subtests should contribute to the total score depending on their actual importance, that is their sensitivity, and not depending on their maximum raw scores. For example, in the word pair associate learning task of the PANDA the maximum score is 12 for the immediate recall and 4 for the delayed recall. However, the delayed recall is a better indicator for cognitive deterioration. Third, the total score should be independent of sociodemographic effects; an age correction is required.

A maximum transformed score of 30 was arbitrarily determined for the PANDA.

Classification rates of the subtests were analyzed and revealed that the verbal fluency task and the delayed recall condition of the word pair associate learning task obtained the highest sensitivity rates (more than 70\%) and that the mental rotation task and the immediate recall of the pair associate learning task were least sensitive (both less than 65\%). To account for these 
differences, the verbal fluency task and the delayed recall were given a maximum of 7 transformed points, the working memory and attention task received 6 , and the spatial imagery and immediate recall of the paired associate learning task received a maximum of 5 transformed points ( 30 points in total).

The second step was to find concrete transformation algorithms of the raw scores on the basis of the CG data and under consideration of the age effects. As such, all algorithms, except for the working memory and attention task (no age effect), were defined for two age groups up to 59 and 60 years or older, respectively. The mean raw score of the corresponding CG (young group, $\mathrm{n}=51$, mean age 52.2 [SD $=6.0$ ] years and older group, $\mathrm{n}=57$, mean age 67.5 [SD $=$ 6.3] years) was given the highest transformed score; scores of at least two SD below were given the lowest transformed score (i.e. 0 points for a percentile equivalent of 2 ) and the values in between assigned accordingly. Few exceptions were made depending on the distribution of the scores.

For the user of the test the transformation procedure is simple. Age-dependant tables for each subtest which display the range of possible raw scores and their corresponding transformed scores are provided. For the final PANDA total score the transformed scores have to be summed up.

With this procedure the mean total PANDA score was $23.6(\mathrm{SD}=5.4)$ in the CG (purified from age effects), $22.7(\mathrm{SD}=4.3)$ for the PD patients, $15.8(\mathrm{SD}=5.0)$ for the PD-MCD group, and $7.9(\mathrm{SD}=5.6)$ for the PDD group. The transformed scores for all subtests and the total PANDA test scores for the study groups are demonstrated in Fig. 3. In a variance analysis significant main effects were found for all transformed subtest scores and the total score for the subtests $(\mathrm{F}(3,226)=$ between 15.8 and 45.5 , all $\mathrm{p}<0.001$, partial $\eta 2=$ between 0.174 and 0.376$)$ and for the total score $(F(3,226)=71.2, p<0.001$, partial $\eta 2=0.486)$. There were no significant differences between the CG and the PD group, and both the CG and PD group showed superior performance compared to the PD-MCD and the PDD patients in all transformed scores $(\mathrm{p}<0.001$ for all group comparisons, except for one significant contrast of the PD versus PD-MCD group in spatial imagery with $p<0.05)$. All transformed scores of the PDMCD group except for the spatial imagery task were higher than those of the PDD patients $(\mathrm{p}<0.001)$.

In a further step, a cut-off score for cognitive impairment was defined. In accordance with established procedures [10], cognitive dysfunction was determined on the basis of the results of the CG, and all scores within one SD below the mean score were regarded as ,age adequate" ( $\geq 18$ points), and scores below 1.5 SD of CG data were determined as indicating clear cognitive disturbance, that is dementia ( $\leq 14$ points). Scores in between one and 1.5 SD below mean CG data were defined to reflect subtle cognitive disturbance (15-17 points). ROC analysis further supported the cut-off score for cognitive impairment of 17/18 points: with this cut-off the PANDA has a specificity of $91 \%$ and a sensitivity of $90 \%$ when including only the PDD patients, and it has a sensitivity of $77 \%$ when both the PD-MCD and the PDD patients are included as a cognitively impaired group. The areas under the curve are 0.917 and 0.708 , respectively. The ,specificity“ for the PD patients that were not cognitively impaired was $89 \%$ (i.e. these patients had scores of 18 points or above). With regard to the lower cut-off score of $14 / 15$ points, $84 \%$ of the PDD patients had PANDA scores below this cut-off. Finally, of all PD patients $52 \%$ subjects were classified to be cognitively impaired (63\% were impaired according to the expert rating). 
In 30 control subjects who were retested after 6 months, the retest reliability for the PANDA cognition part was $r=0.93(p<0.001)$. There was no significant change of the answers in the mood questionnaire $(p=0.253)$. The interrater reliability tested in a subpopulation of 50 PD patients was $r=0.95(p<0.001)$. The cognition part of the PANDA correlates with the MMSE with $r=0.75$ for all subjects, $r=0.48$ for the $C G, r=0.73$ for the PDD patients, and $r=0.76$ for the PD patients (all $\mathrm{p}<0.001$ ). In all PD patients, the PANDA transformed total score of the cognition part correlated significantly with the BDI $(r=-0.34, p=0.001)$.

The median of the mood questionnaire (max. 9) was 2 (range $0-6$ ) in the CG and 3 (range 0 9 ) in the PD group. A ROC analysis was performed with all PD patients and using the BDI classification of patients with or without depressive symptoms (cut-off score of 10/11) as a gold standard [39]. This analysis revealed an optimal cut-off score of 4 points for the PANDA mood questionnaire, showing a good balance of sensitivity $(80 \%)$ and specificity $(92 \%)$. For the whole PD group (all subgroups), a significant correlation of the PANDA mood questionnaire and the BDI ( $\mathrm{rho}=0.64, \mathrm{p}<0.001)$ was observed. The correlations of the PANDA mood questionnaire and the BDI in the study subgroups were rho $=0.65(\mathrm{p}<0.001)$ in the PD group, rho $=0.48(\mathrm{p}<0.01)$ in the PD-MCD group, and rho $=0.68(\mathrm{p}<0.001)$ in the PDD group. The PANDA mood questionnaire correlated with the PANDA cognition transformed total score with rho $=-0.29(\mathrm{p}<0.001)$ for all PD patients.

\section{Discussion}

A detection of cognitive impairment early in the course of PD is useful for the patient, caregiver, and physician to plan future treatment. For example, recent studies suggest that cholinesterase inhibitors may be effective in the treatment of dementia in PD [36]. Our study demonstrates that the new cognitive screening instrument PANDA is an appropriate tool to detect cognitive dysfunction and dementia in Parkinson patients in clinical settings and for general practitioners. It is short (8-10 min), easy to administer, and, following score transformation, is independent of age and education (although education effects might exist in very low-educated populations and are subject to further investigation). With its high construct validity, objectivity, and retest and inter-rater reliability the instrument fulfills relevant psychometric criteria. Most importantly, it has high specificity and high sensitivity. It should be emphasized though that this validation study has been performed in a sample of Parkinson patients and that the reported sensitivity and specificity refer to this specific patient group and not a more general group of older people with possible cognitive dysfunction, or patients with other forms of dementia (e.g. Alzheimer's disease). The PANDA has been developed for PD patients and can be used when PD has been diagnosed. Finally, an important characteristic is the PANDA's well-balanced assessment of cognitive domains typically affected in PD patients i.e. executive functions, memory (strategic encoding and retrieval) and working memory and attention, as well as visuospatial functions. This balance is probably responsible for the PANDA's high sensitivity even for MCD in PD patients. Future studies will have to demonstrate the sensitivity for other frequently used concepts such as MCI in its original form [31] or in later defined subtypes (amnestic versus nonamnestic MCI) [37].

The PANDA thus has some advantages compared to existing other cognitive screening instruments. The MMSE [32] is probably the most frequently used screening tool. However, it has been criticized for lack of sensitivity [38], and education and age dependency [39]. Agespecific norms for the MMSE have been defined [40] but are not integrated in the tool's common test versions and are thus rarely used. Also, the MMSE (and other instruments such as MIS [10]) has been designed for „cortical“ types of dementia which differ from 
„subcortical“" dementia [41] and does not, for example, assess executive functions.

Consequently, our data demonstrate that the MMSE's sensitivity to detect cognitive impairment in PD patients is much lower than that of the PANDA. The ACE [6] comprises basic items of the MMSE and additional short subtests including language, verbal fluency (and thus executive), and visuospatial measures. To our knowledge there are no reports of the use of the ACE in patients with idiopathic PD, and in fact its item composition again may not grasp PD patients0 typical cognitive deficits. Furthermore, performance in the visuoconstructive tasks (copying a two and a three-dimensional figure) may confound with motor dysfunction in these patients. The Mattis dementia rating scale [42] evaluates attention, perseveration, praxis, abstraction, and verbal and nonverbal recent memory. It is frequently used to indicate dementia in patients with $\mathrm{PD}$, but is not a proper tool to detect mild cognitive dysfunction. The Seven Minute Screen [7] has been proved to be sensitive in different types of dementia and also MCI but to the knowledge of the authors has not been specifically tested in PD patients. Performance in this tool depends on age, education and gender, and the administration time may be higher than its name suggests [43]. The new CAMCOG-R battery [44] added two new subtests of executive functions - ideational fluency and visual reasoning - but according to a study by Leeds et al. [45] these tasks seem to offer little advantage, and there are concerns about their construct validity. Furthermore, the scale seems to be inappropriate as a screening tool, due to an administration time of $23 \mathrm{~min}$. Finally, the SCOPA-COG presented by Marinus and colleagues [16] has been developed as a reliable and valid test battery specifically designed to assess cognitive deficits in PD patients. This tool was not designed as a screening tool or a diagnostic instrument but instead is intended for comparing groups in research settings. In summary, the cognitive part of the PANDA fills the gap of an appropriate screening instrument to detect cognitive dysfunctions in PD patients in clinical settings and for general practitioners.

A special further feature of the PANDA is the fact that it accounts for the most frequent neuropsychiatric symptom in PD patients, namely depression. The mood questionnaire corresponds well with the results in the BDI as a ,gold“" standard and can thus be regarded as a valid but short and easy tool for the clinician to objectify possible depressive symptoms. Depression may be present in up to half of the PD patients and is of high clinical relevance [5]. Depression can adversely affect health-related quality of life and can have a significant impact on the patient0s cognitive functions, especially in the domains of memory and attention [46]. Our data also demonstrate this relationship. Thus, when using the PANDA one has to be aware of the fact that cognitive dysfunctions as elicited by this brief instrument may at least in part be confounded with depressive symptoms - and may be modulated by their treatment. However, a decision on whether cognitive dysfunctions are secondarily caused by depression or constitute primary symptoms of the disease is a difficult question per se and can certainly not be answered by using a short screening instrument.

In general it must be emphasized that screening tools are helpful in identifying possible patients with cognitive disturbance. However, they do not replace an elaborate neuropsychological test battery. Further studies with larger patient samples - both demented and non-demented - are desirable to confirm the discrimination rates that were determined on the basis of our data base. Additionally, more research is required that elucidates the relation of the PANDA scores with other neuropsychological test instruments [47]. Furthermore, effects of clinical parameters in PD, such as disease duration and severity, on performance in the PANDA should be examined. Finally, further studies must show the PANDA's capability to reflect treatment effects in the form of cognitive training or cognitive enhancers. 


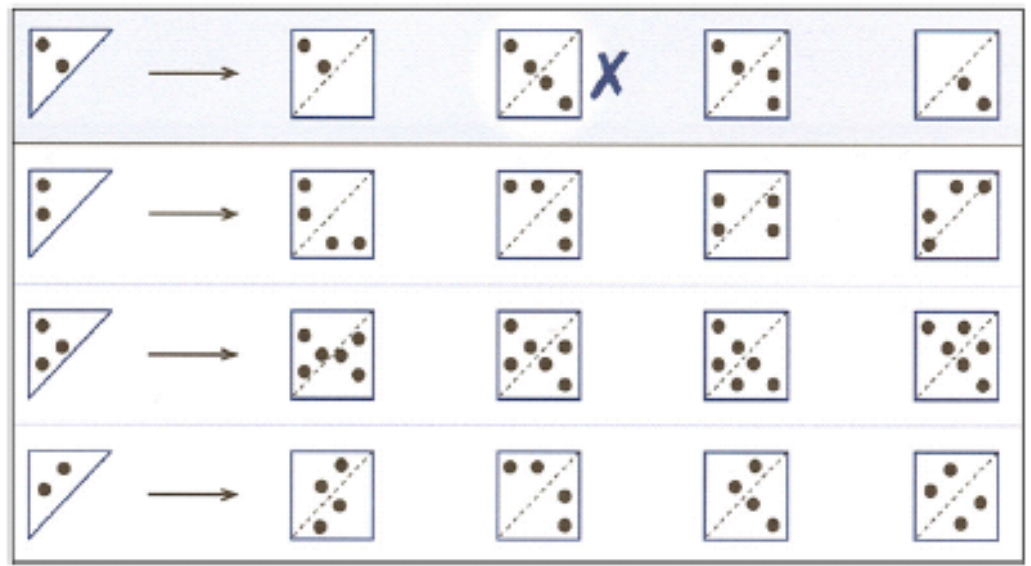

Fig. 1. Items of the spatial imagery task. The kft figure in each fow shows a half-masked square with dot patterns. Out of the three altematives on the right side, the patient is expected to find the pattern which emerges on removing the mask. The upper fow is an example with an " $x$ " marking the correct answer.

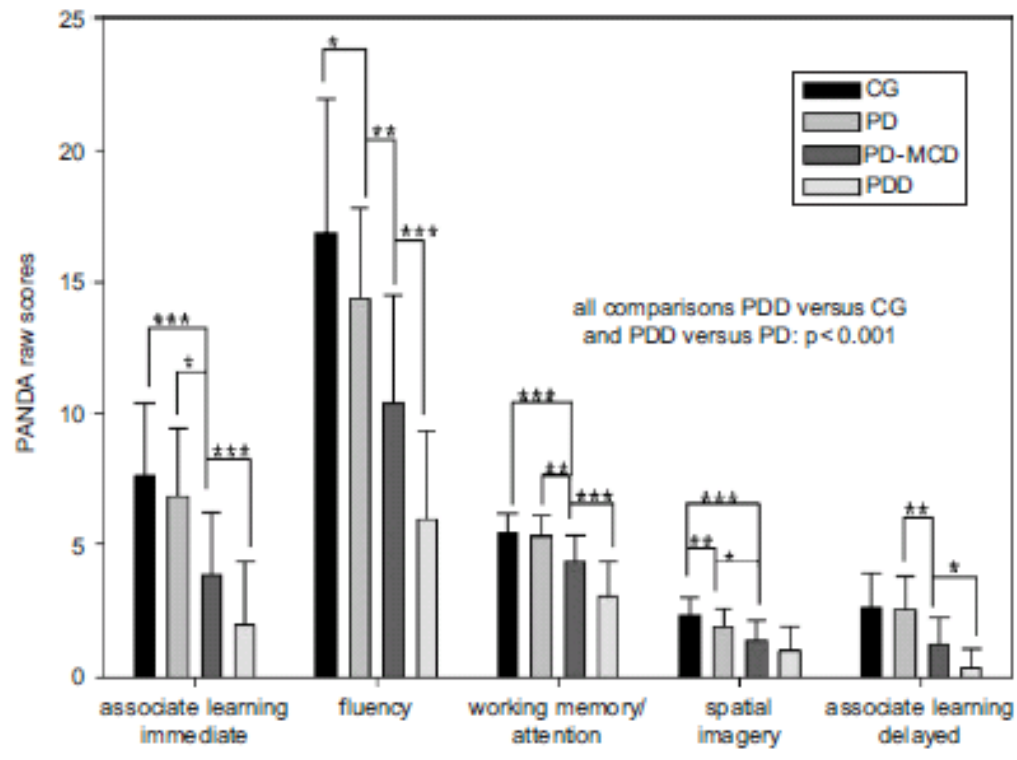

Fig. 2. Raw scores of the PANDA in the study groups. The maximal raw scores of the subtests are associate kaming immediate: 12 , fluency: no maximum, working memory: 6, spatial imagery: 3 , associate leaming delayed: $4 .{ }^{*} p<0.05 ; * * p<0.01 ; * * *<0.001$. 


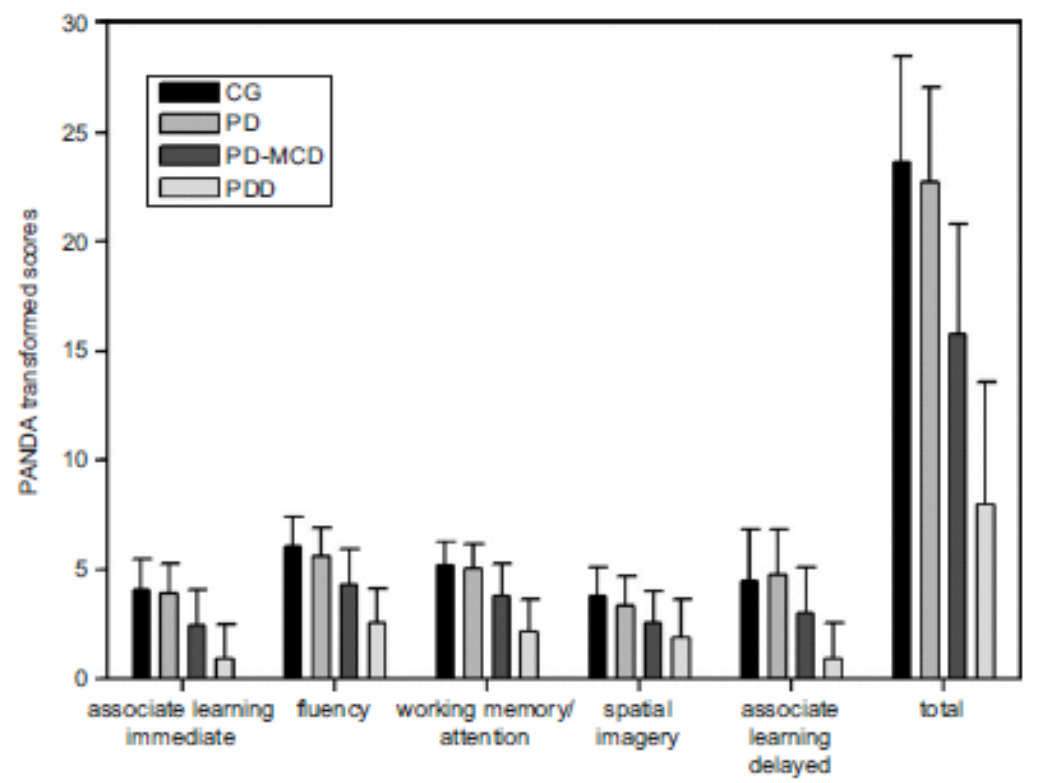

Fig. 3. Transformed PANDA subtest scores (means and standard deviations) of the $\mathrm{CG}$ and PD group. The maximal transformed scores of the subtests are associate leaming immediate: 5, fluency: 7, working memory: 6, spatial imagery: 5, associate learning delayed: 7. The CG and PD groups differed significantly from the PD-MCD and the PDD groups in all subtests (all $p<0.001$ ), and the PD-MCD group was also superior to PDD group (all $p<0.001)$. Only the spatial imagery task did not differ in the PD versus PD-MCD group and the PD-MCD versus PDD group.

Table 1

Sociodemographic and clinical draracteristics of study groups

\begin{tabular}{|c|c|c|c|c|c|}
\hline & $\begin{array}{l}\mathrm{CG} \\
(n=108)\end{array}$ & $\begin{array}{l}\text { PD } \\
(n=46)\end{array}$ & $\begin{array}{l}\text { PD-MCD } \\
(n=40)\end{array}$ & $\begin{array}{l}\text { PDD } \\
(n=38)\end{array}$ & $p$ \\
\hline Age (years): Mean (SD) & $60.3(9.9)$ & $63.9(8.5)$ & $70.2(6.5)$ & $71.1(7.9)$ & $\begin{array}{l}\text { CG:PD-MCD*** } \\
\text { CG:PDD*** } \\
\text { PD:PD-MCD* } \\
\text { PD:PDD** }\end{array}$ \\
\hline $\begin{array}{l}\text { Gender }(\%): \\
\text { Make : Femake }\end{array}$ & $46: 54$ & $50: 50$ & $62: 38$ & $68: 32$ & n.s. \\
\hline Education (years): Mean (SD) & $14.2(2.6)$ & $14.1(2.6)$ & $12.2(2.3)$ & $11.9(3.1)$ & $\begin{array}{l}\text { CG:PD-MCD** } \\
\text { CG:PDD** } \\
\text { PD:PD-MCD* } \\
\text { PD:PDD** }\end{array}$ \\
\hline Disease duration (years): Mean (SD) & - & $6.5(7.3)$ & $10.7(10.2)$ & $8.8(5.7)$ & n.s. \\
\hline $\begin{array}{l}\text { Hoehn \& Yahr stage: } \\
\text { Stage I } \\
\text { Stage II } \\
\text { Stage III } \\
\text { Stage IV } \\
\text { Stage V }\end{array}$ & - & $\begin{array}{l}6 \% \\
30 \% \\
53 \% \\
11 \% \\
0 \%\end{array}$ & $\begin{array}{l}3 \% \\
19 \% \\
53 \% \\
25 \% \\
0 \%\end{array}$ & $\begin{array}{l}3 \% \\
4 \% \\
33 \% \\
47 \% \\
13 \%\end{array}$ & $\begin{array}{l}\text { PD:PDD*** } \\
\text { PD-MCD:PDD** }\end{array}$ \\
\hline MMSE: Mean (SD) & $28.8(1.3)^{2}$ & $28.9(1.0)$ & $27.0(2.2)$ & $20.5(5.9)$ & $\begin{array}{l}\text { CG:PD-MCD** } \\
\text { PD:PD-MCD* } \\
\text { CG:PDD*** } \\
\text { PD:PDD*** } \\
\text { PD-MCD:PDD*** }\end{array}$ \\
\hline BDE: Mean (SD) & $6.7(5.7)^{2}$ & $11.4(8.9)$ & $12.5(7.9)$ & 20.9 (13.3) & $\begin{array}{l}\text { CG:PD-MCD** } \\
\text { CG:PDD*** } \\
\text { PD:PDD** } \\
\text { PD-MCD:PDD*** }\end{array}$ \\
\hline
\end{tabular}

$* p<0.05 ; * *<0.01 ; * * *<<0.001 ;$ n.s.: not significant; only significant gro up comparisons are listed.

"fifty control subjects were tested with the MMSE and the BDI.

\section{Acknowledgments}

This study was funded in part by the EC-FP6-project DiMI, LSHB-CT-2005-512146 and also supported by Novartis Pharma GmbH (Nürnberg, Germany). We thank Katharina Hullmann 
(Parkinsonklinik im Zentrum für Bewegungsstörungen, Bad Nauheim, Germany) and David Emmans and Dr. Gerd Fuchs (Parkinson-Klinik Wolfach, Germany) for arranging the examination of the Parkinson patients.

\section{References}

[1] Bosboom JLW, Stoffers D, Wolters EC. Cognitive dysfunction and dementia in Parkinson's disease. J Neural Transm 2004;111:1303-15.

[2] Braak H, Rüb U, Jansen Steur EN, Del Tredici K, de Vos RA. Cognitive status correlates with neuropathologic stage in Parkinson disease. Neurology 2005;64:1404-10.

[3] Cummings JL. Subcortical dementia. Neuropsychology, neuropsychiatry, and pathophysiology. $\mathrm{Br}$ J Psychiatry 1986;149:682-97.

[4] Emre M. Dementia associated with Parkinson's disease. Lancet Neurol 2003;2:229-37.

[5] McDonald WM, Richard IH, DeLong MR. Prevalence, etiology, and treatment of depression in Parkinson's disease. Biol Psychiatry 2003;54:363-75.

[6] Mathuranath PS, Nestor PJ, Berrios GE, Rakowicz W, Hodges JR. A brief cognitive test battery to differentiate Alzheimer's disease and frontotemporal dementia. Neurology 2000;55:1613-20.

[7] Solomon PR, Hirschoff A, Kelly B, Relin M, Brush M, et al. A 7 minute neurocognitive screening battery highly sensitive to Alzheimer's disease. Arch Neurol 1998;55:349-55.

[8] Saint-Cyr JA, Taylor AE, Lang AE. Procedural learning and neostriatal dysfunction in man. Brain 1988;111:941-59.

[9] Pillon B, Deweer B, Agid Y, Dubois B. Explicit memory in Alzheimer's, Huntington's, and Parkinson's diseases. Arch Neurol 1993;50:374-9.

[10] Lezak MD, Howieson DB, Loring DW. Neuropsychological assessment. 4th ed. New York: Oxford University Press; 2004.

[11] Cerhan JH, Ivnik RJ, Smith GE, Tangalos EC, Petersen RD, Boeve BF. Diagnostic utility of letter fluency, category fluency, and fluency difference scores in Alzheimer's disease. Clin Neuropsychol 2002;16:35-42.

[12] Monsch AU, Bondi MW, Butters N, Paulsen JS, Salmon DP, Brugger P, et al. A comparison of category and letter fluency in Alzheimer's and Huntington's disease. Neuropsychology 1994;8:25-30.

[13] Kessler J, Bley M, Mielke R, Kalbe E. Strategies and structures in verbal fluency tasks in patients with Alzheimer's disease. Alzheimer's Res 1997;3:59-62.

[14] Henry JD, Crawford JR. Verbal fluency deficits in Parkinson's disease: a meta-analysis. J Int Neuropsychol Soc 2004;10:608-22.

[15] Hovestadt A, de Jong GJ, Meerwaldt JD. Spatial disorientation as an early symptom of Parkinson's disease. Neurology 1987;37:485-7.

[16] Waterfall ML, Crowe SF. Meta-analytic comparison of the components of visual cognition in Parkinson's disease. J Clin Exp Neuropsychal 1995;17:759-72.

[17] Crucian GP, Barrett AM, Burks DW, Riesttra AR, Roth HL, Schwartz RL, et al. Mental object rotation in Parkinson's disease. J Int Neuropsychol Soc 2003;9:1078-87.

[18] Lee AC, Harris JP, Calvert JE. Impairments of mental rotation in Parkinson's disease.

Neuropsychologia 1998;36:109-14.

[19] Antal A, Bandini F, Keri S, Bodis-Wollner I. Visuo-cognitive dysfunctions in Parkinson's disease. Clin Neurosci 1998;5:147-52.

[20] Girotti F, Soliveri P, Carella F, Piccolo I, Caffarra P, Musicco M, et al. Dementia and cognitive impairment in Parkinson's disease. J Neurol Neurosurg Psychiatr 1988;51:1498-502.

[21] Baddeley AD. Is working memory still working? Am Psychol 2001; 56:851-64.

[22] Gilbert B, Belleville S, Bherer L, Chouinard S. Study of verbal working memory in patients with Parkinson's disease. Neuropsychology 2005;19:106-14.

[23] Higginson CI, King DS, Levine D, Wheelock VL, Khamphay NO, Sigvardt K. The relationship between executive function and verbal memory in Parkinson's disease. Brain Cogn 2003;52:343-52.

[24] Kemps E, Szmalec A, Vandierendonck A, Crevits L. Visuo-spatial processing in Parkinson's disease: evidence for diminished visuospatial sketch pad and central executive resources.

Parkinsonism Relat Disord 2005;11:181-6. 
[25] Bronnick K, Ehrt U, Emre M, De Deyn PP, Wesnes K, Tekin S, et al. Attentional deficits affect activities of daily living in dementiaassociated with Parkinson's disease. J Neurol Neurosurg Psychiatry 2006;77:1136-42.

[26] Hughes CP, Berg L, Danziger WL, Coben LA, Martin RL. A new clinical scale for the staging of dementia. Br J Psychiatry 1982;140:566-72.

[27] Hughes AJ, Daniel SE, Kilford L, Lees AJ. Accuracy of clinical diagnosis of idiopathic Parkinson's disease: a clinico-pathological study of 100 cases. J Neurol Neurosurg Psychiatry 1992;55:181-4.

[28] American Psychiatric Association. Diagnostic and statistical manual of mental disorders (DSMIV), 4th ed. Washington, DC: Author; 1994.

[29] World Health Organisation. International statistical classification of diseases and health related problems. Tenth version (ICD-10). Geneva: Author; 1994.

[30] Christensen H, Henderson AS, Jorm AF, Mackinnon AJ, Scott R, Kotren A. ICD-10 mild cognitive disorder: epidemiological evidence on its validity. Psychol Med 1995;25:105-20.

[31] Petersen RC, Smith GE, Waring SC, Ivnik RJ, Tangalos EG, Kokmen E. Mild cognitive impairment: clinical characterization and outcome. Arch Neurol 1999;56:303-8.

[32] Folstein MS, Folstein SE, McHugh PR. Mini-mental-state - a practical method for grading the cognitive state of patients for the clinician. J Psychiatr Res 1975;12:189-98.

[33] Kessler J, Markowitsch HJ, Denzler P. Der Mini Mental Status Test. Weinheim: Beltz-TestVerlag; 1990.

[34] Beck AT, Hautzinger M, Bailer M, Worall H, Keller F. Beck-Depressions-Inventar (BDI). Göttingen: Hogrefe; 1995.

[35] Monsch AU, Foldi NS, Ermini-Funfschilling DE, Berres M, Taylor KI, Seifritz E, et al. Improving the diagnostic accuracy of the mini mental state examination. Acta Neurol Scand 1995;92:145-50.

[36] Emre M, Aarsland D, Albanese A, Byrne EJ, Deuschl G, De Deyn PP, et al. Rivastigmine for dementia associated with Parkinson's disease. N Engl J Med 2004;351:2509-18.

[37] Petersen RC. Mild cognitive impairment as a diagnostic entity. J Intern Med 2004;256:183-94.

[38] Tierney MC, Szalai JP, Dunn E, Geslani D, McDowell I. Prediction of probable Alzheimer disease in patients with symptoms suggestive of memory impairment. Value of the mini-mental state examination. Arch Fam Med 2000;9:527-32.

[39] Tombaugh TN, McIntyre NJ. The mini-mental state examination: a comprehensive review. J Am Geriatr Soc 1992;40:922-35.

[40] Bleecker ML, Bolla-Wilson K, Kawas C, Agnew J. Age-specific norms for the mini-mental state exam. Neurology 1988;38:1565-8.

[41] Noe E, Marder K, Bell KL, Jacobs DM, Manly JJ, Stern Y. Comparison of dementia with Lewy bodies to Alzheimer's disease and Parkinson's disease with dementia. Mov Disord 2004;19:60-7.

[42] Mattis S. Dementia rating scale: professional manual. Odessa, Fl: Psychological Assessment Resources, Inc.; 1988.

[43] Meulen EF, Schmand B, van Campen JP, de Koning SJ, Ponds RW, Scheltens P, et al. The seven minute screen: a neurocognitive screening test highly sensitive to various types of dementia. J Neurol Neurosurg Psychiatry 2004;75:700-5.

[44] Roth M, Huppert F, Mountjoy C, Tym E. The Cambridge Examination for Mental Disorders of the Elderly—Revised. Cambridge: Cambridge University Press; 1999.

[45] Leeds L, Meara RJ, Woods R, Hobson JP. A comparison of the new executive functioning domains of the CAMCOG-R with existing tests of executive function in elderly stroke survivors. Age Ageing 2001;30:251-4.

[46] Norman S, Troster AI, Fields JA, Brooks R. Effects of depression and Parkinson's disease on cognitive functioning. J Neuropsychiatry Clin Neurosci 2002;14:31-6.

[47] Marinus J, Visser M, Verwey NA, Verhey FR, Middelkoop HA, Stiggelbout AM, et al. Assessment of cognition in Parkinson's disease. Neurology 2003;61:1222-8. 